\title{
' $Y$ ' Shaped right Parotid duct - a rare anatomical presentation
}

\author{
Mohd.Saleem Itoo ${ }^{1 *}$, Raj Tajamul Hussain ${ }^{2}$, Omer Bashir Itoo ${ }^{2}$, Shaheen Shahdad ${ }^{3}$, Mohd.Muzzaffar Lone ${ }^{3}$, \\ Fahmida Akhter ${ }^{2}$, Gh.Mohd Bhat ${ }^{1}$, Bashir Ahmad Shah ${ }^{1}$, Shabir Ahmad Shabir ${ }^{4}$ \\ ${ }^{1}$ Associate Professor Postgraduate Department of Anatomy Government Medical College, Srinagar J\&K, India \\ ${ }^{2}$ Assistant surgeon Postgraduate Department of Anatomy /Medicine, Government Medical College Srinagar, J\&K, India \\ ${ }^{3}$ Professor Postgraduate Department of Anatomy Government Medical College Srinagar, J\&K, India \\ ${ }^{4}$ Lecturer Department of Anaesthesia and Critical care, Government Medical College Srinagar, J\&K, India \\ *Corresponding author E-mail: dr.saleem68@gmail.com
}

Copyright () 2015 Mohd.Saleem Itoo et al. This is an open access article distributed under the Creative Commons Attribution License, which permits unrestricted use, distribution, and reproduction in any medium, provided the original work is properly cited.

\begin{abstract}
The parotid gland with two ducts is a rare anomaly and very few cases have been reported in the literature. The Present study was conducted in the postgraduate Department of Anatomy at Government Medical College Srinagar to study the variations in the pattern and number of ducts of parotid gland during routine dissection for academic purposes between 2001 -20014. Both parotid glands of 36 Indian cadavers were dissected out for routine anatomic teaching and simultaneously recording observations. In this study we also included 10 formalin preserved specimens of parotid gland. It was observed that in one of the cadavers two ducts emerged from the anterior border of right parotid gland. These ducts D1 (superior) and D2 (inferior) joined after crossing the anterior border of the masseter muscle and formed a common duct (D3) in the form of letter ' $\mathrm{Y}$ ', which pierced the buccal pad of fat, buccopharngeal fascia, buccinator muscle and mucous membrane before opening inside the vestibule of mouth. This anomalous duct pattern though rare is of great academic and clinical significance in Otorhinology, Maxillofacial and Plastic surgery, where it may explain the occurrence of post traumatic/iatrogenic parotid fistulas and need for preoperative sialography during surgical procedures of this region.
\end{abstract}

Keywords: Auriculotemporal Nerve; Buccinator Muscle; Buccophryngeal Facia; Frey’s Syndrome; Parotid Gland; Stenson's Duct.

\section{Introduction}

The Parotid gland being the largest and most important salivary gland is an outgrowth from the mouth with an average weight of 25 grams. In 20 percent of individuals an accessory parotid gland is found between parotid duct and zygomatic arch. Its serous secretions are drained by the parotid duct (Stenson's duct). This duct begins by the confluence of two main tributaries within the anterior part of the parotid gland. Emanating at the anterior border of the upper part of the gland it passes horizontally across masseter approximately at the level midway between the angle of the mouth and the zygomatic arch. The average length of parotid duct is $5 \mathrm{~cm}$ [1-8]. The lumen of parotid duct is $3 \mathrm{~mm}$ wide [1] but very narrow near its termination at parotid papilla. After emanating the gland from its anterior border along with branches of facial nerve, it runs over the masseter muscle guarded by upper and lower marginal mandibular branches of the facial nerve about a finger breadth below the zygomatic arch [3]. The parotid duct which is comparatively a superficial structure may get damaged during surgery or trauma. At the anterior margin of this muscle, the duct turns sharply medially almost at right angle and enters into the vestibule of oral cavity after piercing buccal pad of fat, buccopharyngeal fascia, buccinater muscle and the linning mucosa of oral cavity. It opens upon a small papilla (Parotid Papilla) opposite the crown of second maxillary molar tooth [1-8].The oblique course of the duct between the buccinators and the oral mucosa acts as a valve, which prevents the distension of the parotid duct during forceful blowing [3-4]. During its passage over masseter it occasionally receives accessory parotid duct [1] from accessory parotid gland if the same is present .On the surface the parotid duct is represented by middle third of a line drawn from 
the lower border of tragus to the point between the ala of the nose and the red margin of the lip. The resistant cases of chronic recurrent parotitis are treated by injecting antibiotics through the oral aperture of the duct.

Parotid sialography is a radiological procedure for visualizing parotid duct and its branching pattern. It is indicated in cases where obstruction or narrowing of the main parotid duct or of its intraglandular ramifications are suspected. The contrast medium is injected by a small cannula into the oral opening (papilla) of this duct and a radiograph is taken.

The parotid gland is ectodermal in origin. It arises from buccal ectoderm as an outgrowth, which gives rise to the parotid duct and the gland. The site of origin of the outgrowth is retained as the oral orifice of the duct. The sialographic (lateral sialogram) studies of parotid duct show that it is formed near the centre of the posterior border of mandibular ramus by the union of two or three ducts which ascend and descend respectively at right angles to the main duct [1]. As the main duct crosses over the masseter, it receives from above five or six ductules from the accessory parotid gland which when present lie between the duct below and the zygomatic arch above. As it curves round the anterior border of the masseter muscle it is often compressed and its shadow is attenuated. The parotid duct develops as consequence of epithelial -mesenchymal interactions between the ectodermal epithelial lining of the oral cavity and the adjacent neural crest derived mesenchyme. An elongated furrow running dorsally from the angle of mouth between the mandibular and maxillary prominences appears at stage $15(8 \mathrm{~mm})$ which is subsequently converted into a tube which persists as parotid duct. The secretory part of the parotid gland is derived from epithelial cells sprouting from the terminal ramifications of the parotid duct within the substance of gland.

\section{Materials and methods}

This present study was carried out in the Postgraduate department of Anatomy at Government Medical College Srinagar, Kashmir, J\&K, India by dissecting 36 formalin preserved cadavers (33 males and 3 females) over a period of 14 years (2001-2014) for routine teaching purposes and recording observations. Ten dissected specimens of the parotid gland were analyzed and added to this study. Dissection was meticulously carried out strictly following instructions given in the Cunningham's manual of practical Anatomy. Observations regarding the anatomical pattern of the parotid duct were recorded.Vernier Caliper and thread was used to measure the dimensions of these ducts. The length of D1 (Superior duct) and D2 (inferior duct) were taken from anterior border of parotid gland till they united and formed a common duct (D3). The length of D3 was taken from its beginning to its termination. The transverse diameter (outer wall to outer wall thickness) of D1 and D2 was taken $5 \mathrm{~mm}$ distal to the posterior border of masseter and that of D3 was taken $5 \mathrm{~mm}$ from its anterior border. The parotid gland was also dissected out to see the ramification pattern of D1 and D2 within the gland. Appropriate photographs were taken and labeled as shown in fig, [1] and fig, [2].

\section{Results}

During routine dissection of 36 cadavers (n:72 parotid glands) between 2001-2014 and analysis of ten specimens of head and neck, we observed that in one middle aged cadaver, two parotid ducts emerged from the anterior border of the right parotid gland $6 \mathrm{~mm}$ apart. These two ducts, the superior (D1) and the inferior (D2) united with each other near the anterior border of masseter and formed a common duct (D3). This pattern resembled the English letter ' $\mathrm{Y}$ ' as shown in fig, [1] and we designated it as 'Y' pattern of the parotid duct and the two small limbs (D1) and (D2) as "Itoo's" ducts after the Sir name of our first author. The common duct (D3), so formed pierced buccal pad of fat, buccopharyngeal fascia, and buccinators muscle to enter into the vestibule of mouth. The length of D1, D2 and D3 was $21 \mathrm{~mm}, 23 \mathrm{~mm}$ and $44 \mathrm{~mm}$ respectively. The outer wall to wall diameter of D1, D2 and D3 was 3mm, 3mm and 4mm respectively. We traced D1 and D2 proximally even within the substance of parotid gland for 10mm and found that the distance between these two ducts at this site was $9 \mathrm{~mm}$. The length $(5 \mathrm{~cm})$ and anatomical course of the parotid duct on the left side of this cadaver was normal as shown in fig, [2].

\section{Discussion}

There are very few reports in the literature about the variations in the length, course, ramification pattern and modes of termination of the parotid ducts [1-8]. These variations though rare, are likely to encounter during surgical procedures on parotid gland, dissection and at autopsy. A thorough anatomical knowledge of these variations is very useful for Plastic and Maxillofacial surgeons, Otorrhinologists and Radiologists. A sound knowledge of these rare anatomical variations besides helping us in solving many clinical problems like formation of iatrogenic parotid fistulas may also help us in their effective surgical management. Only a few earlier workers have reported about the anatomical variations of parotid ducts. Aktan et al. [9] found a double parotid duct on the right side of a cadaver. The two ducts united and formed a single duct proximal to buccinators. They considered these ducts as extension of the collecting ducts which should have normally united inside the substance of parotid gland and emerged as a single duct. Similar right sided ductal pattern was observed by Fernandes et al. [10] but he failed to trace the origins of these ducts within the parotid gland. According to Bailey the incidence of double parotid duct is $7 \%$ in general population [11]. Rajesh B 
Astik (2011) [12] reported a case of bilateral double parotid duct in a 50-year old male cadaver. On both sides these ducts united near the anterior border of the masseter muscle and formed a single duct which opened into the vestibule of mouth.

In our series we found that on the right side of a middle aged cadaver two parotid ducts (D1 and D2) emerged from the anterior border of the parotid gland $6 \mathrm{~mm}$ apart. These ducts united near the anterior border of masseter muscle and formed a common duct $44 \mathrm{~mm}$ long. On tracing D1 and D2 inside the parotid gland we observed that the distance between these ducts increased from $6 \mathrm{~mm}$ near anterior border to $9 \mathrm{~mm}$, about $1 \mathrm{~cm}$ proximal to anterior border and these ducts (D1 and D2) in reality continued proximally inside the parotid gland. Our observations are similar to those made by earlier workers [8], [9], [10] but they failed to trace these ducts (D1 and D2) inside the gland. The anatomical pattern of the parotid duct reported by us was similar to that reported by Astik in 2011 but in his study he reports it to be present bilaterally in contrast to our unilateral observations. Our observations are also discordant to those made by Baily who reports the incidence of double parotid duct on a higher side [12].

\section{Summary and conclusion}

The anomalies of the parotid duct though very rare have definite embryological basis. The variations in the length and the pattern of this duct are not reported in the literature [1-7]. The main parotid duct is formed within the substance of parotid gland by union of two or more ducts. The parotid duct makes its exit from the middle of its anterior border.

The intraparotid ducts may fail to join within the substance of parotid gland and leave through the anterior border as two separate ducts, which may join at a variable distance from the anterior border of the parotid gland to form a common duct in the form of letter ' $Y$ '. This pattern of the parotid duct does not find its mention in the available literature. The anatomical relations of the ' $\mathrm{Y}$ ' shaped duct with the branches of facial nerve and transverse facial artery are also variable. Thus a sound knowledge of these anomalies is essential for prevention and surgical management of injuries and their sequelae like parotid fistula formation and facial nerve palsy. The variations in the anatomical pattern of the parotid duct also justify need for preoperative sialography in patients who undergo surgery in parotid and maxillofacial region.

\section{Figure Legends}

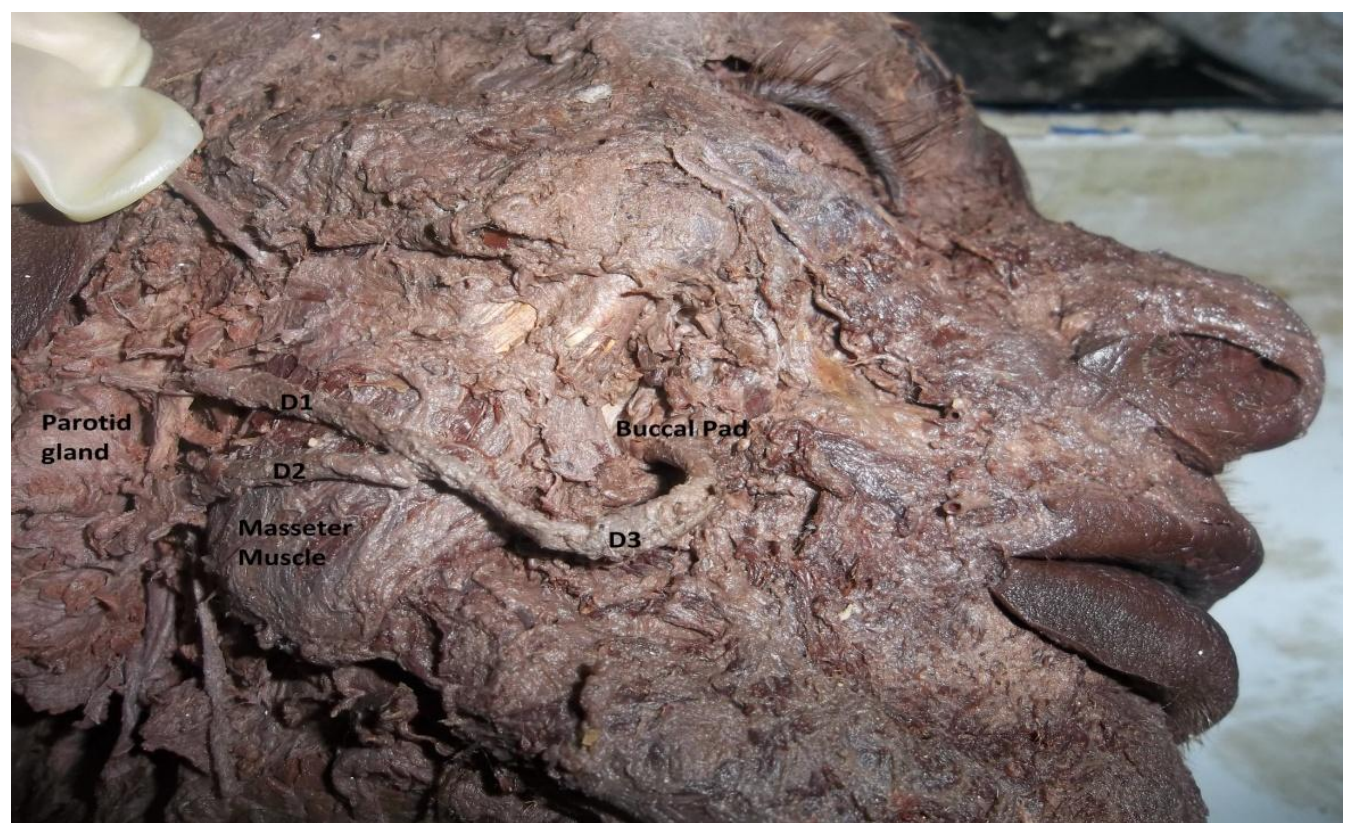

Fig. 1: 'Y' Shaped right parotid duct, Two ducts, superior (D1) and Inferior (D2) emanating from the anterior border of the right Parotid Gland. These ducts join to form a Common duct (D3) near the anterior border of masseter muscle. The common duct then Pierces buccal Pad of fat, buccophryngeal fascia and buccinators muscle to open in the vestibule of mouth opposite the crown of Second maxillary molar tooth. 


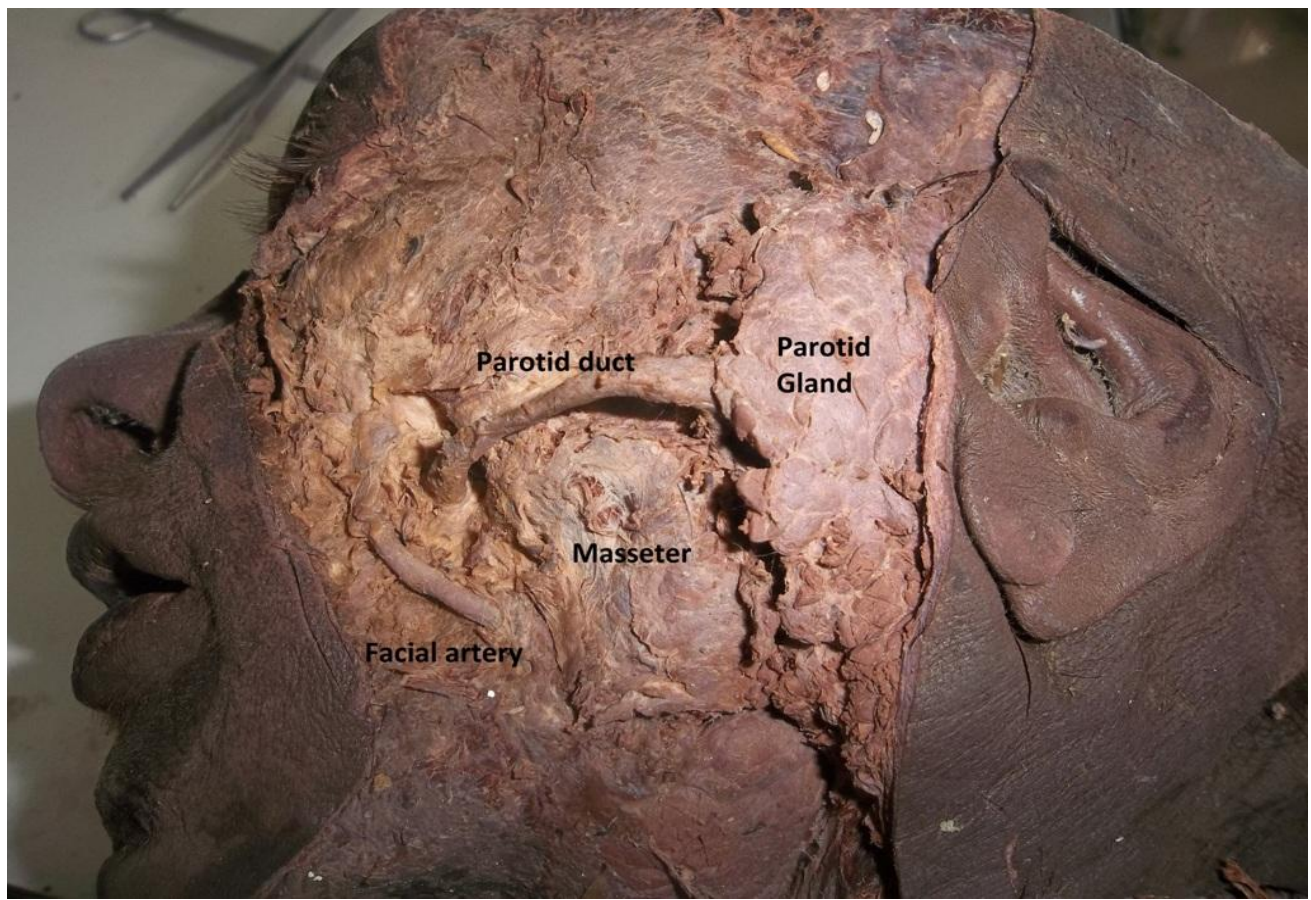

Fig. 2: Normal Left Parotid duct of the same Cadaver emerging from the anterior border of parotid gland.

\section{References}

[1] Gray's Anatomy;.The Anatomical Basis Of Medcine And Surgery. 38th Ed. Elsevier Churchill Living Stone. 2000; Pp: 1691.

[2] Gray' Anatomy; The Anatomical Basis Of Clinical Practice.39th Ed. Elsevier Churchill Living Stone. 2005; Pp: 516.

[3] Richards. Snell; Clinical Anatomy, 7th Edition, Lippincott Williams \& Wilkins.2004; Pp 773.

[4] Neeta V Kulkarni; Clinical Anatomy.2nd Edition, Jaypee Brothers Medical Publishers (P) Ltd New Delhi. 2012; Pp 340-41.

[5] Dk Kadasne Textbook of Anatomy (Volume 3). Ist Edition 2009, Jaypee Brothers Medical Publishers;Pp 826.

[6] James L. Hiatt, Leslie P.Gartner; Text Book of Head and Neck Anatomy 1982, Appleton-Centuary-Crafts/New York.Pp; 191.

[7] B D Chaurasia. Human Anatomy, 4th Edition,Volume 3. C B S Publishers \& Distributors New Delhi 2004. Pp; 136-37.

[8] Keith \& Moore. Clinically Oriented Anatomy 1980,Williams \& Wilkins Baltimore/London 1980.Pp; 895

[9] Aktan Za,Bilge O,Pinar Ya,Ikiz Ao. Duplication of the Parotid Duct: A Previously Unreported Anomaly.Surg Radiol Anat.2001; 23:353-354. Http://Dx.Doi.Org/10.1007/S00276-001-0353-Y.

[10] Fenandes ACS, Lima Gr, Rossi Am, Aguiar Cm. Parotid Gland with Double Duct: An Anatomic Variation Description.Int J Morphol.2009; 27:129-132.

[11] Baily L. Short Practice of Surgery.15th Ed.London, Lewis.1971:533.

[12] Rajesh B Astik.Embryological Basis of Bilateral Double Parotid Ducts: A Rare Anatomical Variation. 